\title{
Frequency of smoking cessation interventions from health care providers in Minnesota
}

\author{
Megan N. Whittet ${ }^{1^{*}}$, Raymond G. Boyle ${ }^{1}$, Jeong Kyu Lee ${ }^{1}$, Ann W. St. Claire $^{1}$, Joanne D'Silva ${ }^{1}$, \\ Peter Rode ${ }^{2}$, Ann M. Kinney ${ }^{2}$
}

${ }^{1}$ Research Department, ClearWay Minnesota ${ }^{\mathrm{SM}}$, Minneapolis, USA; *Corresponding Author: mwhittet@clearwaymn.org

${ }^{2}$ Center for Health Statistics, Minnesota Department of Health, St. Paul, USA

Received 29 February 2012; revised 4 March 2012; accepted 15 March 2012

\begin{abstract}
Objective: There is strong evidence supporting the impact of health care provider advice on patients' smoking behavior. This paper examines adult smoker reports of health care provider smoking cessation interventions. Design: The 2010 Minnesota Adult Tobacco Survey (MATS) is a random digit dial telephone survey with 7057 Minnesota adults. Both landline and mobile telephone samples were included. These samples were combined and weighted to represent the population of adults in Minnesota. Results: Among adult Minnesotans, $16.1 \%$ were current smokers, $80.9 \%$ reported seeing a health care provider in the past 12 months and $80.2 \%$ of those adults were asked if they smoke. Among smokers who saw any provider in the past 12 months, $94.4 \%$ reported they were asked about smoking and $71.8 \%$ were advised not to smoke. In contrast, $43.9 \%$ of smokers received referrals from a health care provider and among those, $37.5 \%$ were recommended medications and $24.8 \%$ were referred to a quit smoking program. Conclusion: Given the large body of evidence supporting cessation interventions by health care providers, the increase in use of electronic medical records and the availability and success of evidence-based cessation programs, efforts to improve rates of identifying and treating each tobacco user are needed. Further research is needed to explore how cessation intervention responsibilities can be shared among the clinical team and integrated into ongoing clinical care.
\end{abstract}

Keywords: Smoking Cessation; Survey Research; Guideline; Health Care Provider

\section{INTRODUCTION}

Smoking remains the number one preventable cause of death and disease in the United States, causing approximately 443,000 deaths each year and a national economic burden of over $\$ 193$ billion dollars annually in health care costs and lost productivity [1]. This is despite the fact that evidence-based interventions exist that can be efficiently delivered to a vast majority of smokers.

Health care providers are uniquely positioned to help smokers quit smoking. The majority of adults see a health care provider at least once each year [2] and approximately $70 \%$ of smokers are seen by a clinician each year [3]. A wide variety of providers such as physicians, nurses, dentists, hygienists, pharmacists and medical assistants can effectively implement the brief evidencebased treatment interventions outlined in the Public Health Service Clinical Practice Guideline (the Guideline) [4]. The core components of the Guideline's recommendation, often referred to as the 5A's, include: asking every patient if they use tobacco, advising tobacco users to quit and assessing their willingness to make a quit attempt. Those willing to make a quit attempt should be assisted by providing medication and counseling. Those unwilling to make quit attempts should be encouraged with a brief motivational discussion. Follow-up should be arranged at the next visit [4]. A large body of evidence has accumulated over the past 15 years demonstrating the impact of this approach in the health care setting [4].

The effectiveness of this intervention-based assessment has been well accepted. A Cochrane review of eleven studies found that health care providers offering advice to stop smoking increased long-term abstinence by up to $47 \%$, and offering assistance doubled quit attempts [5]. Furthermore, the effects of health care provider advice alone on patients' smoking, quit rates and long-term abstinence rates are significant [4-7]. For instance, the Florida Tobacco Callback Survey found that smokers are about $50 \%$ more likely to attempt to quit smoking, both in their lifetime and recently, if they receive advice to quit from a health care provider [6]. 
Nonetheless, the implementation of these recommended clinical practices is less than optimal. Numerous barriers, in particular time, have been noted and studies have found differences in the frequency of providing advice based on smokers' level of dependence, demographic characteristics and/or health status. One study reported that heavier smokers were twice as likely as light smokers to be offered and to use pharmacotherapy [8]. Additional studies have found variation in health care provider advice to quit based on patient income, education and health status $[9,10]$.

However, much of the research describing the implementation of the smoking cessation Guideline was completed when smoking prevalence rates were considerably higher and the presence of electronic medical record (EMR) systems that might facilitate provider intervention behaviors was much lower. According to the 2011 National Ambulatory Medical Care survey, adoption of any EMR system has increased to an average of $57 \%$ among office-based physicians and is as high as $84 \%$ in North Dakota [11]. In Minnesota, the reported rate of any EMR use was $78 \%$ in 2011 [11].

This paper examined recent statewide surveillance data to: 1) determine the frequency that health care providers in Minnesota are intervening with smoking patients; 2) determine the differences in intervening based on patient characteristics; and 3) examine changes between 2007 and 2010. Given the large amount of evidence supporting the Guideline and the increase in the use of EMR systems, it was hypothesized that there would be an increase in asking, advising, and assisting and referring patients to evidence-based cessation services.

\section{METHODS}

\subsection{Data Source}

The Minnesota Adult Tobacco Survey (MATS) is a cross-sectional, random digit dial (RDD) telephone survey, designed to collect data about tobacco use and attitudes from a representative sample of adults aged 18 years and older living in Minnesota. MATS 2010 used a list-assisted RDD sampling method to survey a total of 7057 adults, including 5555 from a statewide landline sample and 1502 from a cell phone sample. Data collection took place between February and May of 2010. MATS 2007 had a total sample of 12,580 adults. The same vendor used common methods in both 2007 and 2010 to conduct the survey such as, computer assisted telephone interviewing and consistent core questions. MATS data was weighted to represent the entire noninstitutionalized adult population in Minnesota. There are some differences in the survey samples between the two years. In 2010 a cell phone component to the sample was included. In 2007 the sample included members from
BlueCross and BlueShield of Minnesota and an over sample of African Americans and young adults. Although there were differences in the sampling, 2007 and 2010 are comparable population-based samples.

Methodology developed by the American Association for Public Opinion Research was used to calculate the weighted response rate for the 2010 landline sample $(45.0 \%)$ and the cell phone sample (44.5\%) [12]. The response rate for 2007 was $40.7 \%$ [13]. These response rates are comparable to other large RDD surveys, such as the Behavioral Risk Factor Surveillance System survey [14]. More information on the methods for the 2010 MATS survey can be found in the complete report, Tobacco Use in Minnesota: 2010 Update and the 2007 report, Creating a Healthier Minnesota: Progress in Reducing Tobacco Use. Both reports are available at www.mnadulttobaccosurvey.org.

The Minnesota Department of Health Institutional Review Board reviewed and approved the 2007 and 2010 MATS questionnaire, data collection and data security procedures.

\subsection{Measures}

For the MATS, an adult current smoker was defined as a person aged $\geq 18$ years who has smoked $\geq 100$ cigarettes in his/her lifetime and now smokes every day or some days. A former smoker was defined as someone who has smoked $\geq 100$ cigarettes in his or her lifetime but does not currently smoke at all, and a never smoker was someone who has not smoked at least 100 cigarettes in his or her lifetime. In addition to assessing smoking frequency, current smokers were asked how many cigarettes they smoke on average on the days that they smoke.

Respondents were asked if they had visited any type of health care provider in the past 12 months. If the response to this question was yes, the MATS survey inquired about three of the five core components of the Guideline: asking about smoking status, advising patients to quit and assisting them by referring smoking cessation treatment or services (see Table 1 for survey questions).

To assess health status, respondents were asked to rate their health as "excellent", "very good", "good", "fair" or "poor". Respondents also reported if they had any type of health insurance over the past 12 months.

\subsection{Data Analysis}

Statistical analyses were conducted using SPSS version 19.0 with the Complex Samples module. Bivariate techniques were utilized to test if provider intervention rates differed by demographic characteristics, smoking status and smoking frequency. Specifically, statistical differences in the proportions were assessed at 0.05 alpha level based on the z-distribution. 
Table 1. Survey questions to examine the ask, advise and assist/refer intervention.

\begin{tabular}{ll}
\hline Ask & $\begin{array}{l}\text { During the past } 12 \text { months, did any doctor, nurse, } \\
\text { dentist, pharmacist, or any other kind of health } \\
\text { professional ask if you smoke? }\end{array}$ \\
Advise & $\begin{array}{l}\text { During the past } 12 \text { months, did any doctor, nurse, } \\
\text { dentist, pharmacist, or any other kind of health }\end{array}$ \\
professional advise you to quit smoking? & \\
Assist/Refer & Three consecutive questions: \\
& 1) In the past 12 months, did any of these health \\
professionals you saw... recommend any product or \\
prescription for a medication to help you quit \\
smoking? \\
2) In the past 12 months, did any of these health \\
professionals you saw... suggest that you seek help \\
to quit smoking using a quit smoking program, such \\
as a helpline, a class or group or an online website or \\
program? \\
3) In the past 12 months, did any of these health \\
professionals you saw help you access that quit \\
smoking program?
\end{tabular}

\section{RESULTS}

The prevalence rate in Minnesota in 2007 was 17.0\% and in 2010 the rate was $16.1 \%$ [12]. Despite no change in the overall smoking prevalence the percentage of current smokers who smoke 25 or more cigarettes per day decreased from $10.3 \%$ in 2007 to $6.3 \%$ in 2010 (p < 0.05 ) [12].

\subsection{Health Care Provider Visits}

Among all adult Minnesotans, $80.9 \%$ reported seeing a health care provider in the past 12 months. Table 2 presents demographic characteristics for those who visited a health care provider in the past 12 months among current, former and never smokers. Former smokers were the most likely to have had a health care provider visit (87.6\%) followed by never smokers $(80.7 \%)$ and current smokers $(70.0 \%)$. Each pairwise comparison was significant at $\mathrm{p}<0.05$. Females saw a health care provider at a higher rate than males regardless of smoking status, and current female smokers were significantly more likely to have had a health care visit compared to male smokers $(80.8 \%$ vs. $61 \%, \mathrm{p}<0.05)$. In addition, smokers with at least some college education were more likely to have seen a health care provider than smokers with a high school degree $(77.1 \%$ vs. $61.2 \%, \mathrm{p}<0.05)$. This was not the case for former or never smokers.

Among all adults who saw a health care provider in the past 12 months, $80.2 \%$ reported being asked if they smoke. Of the current smokers who saw a health care provider, $94.4 \%$ reported being asked if they smoke and $71.8 \%$ reported being advised to quit. However, fewer than half $(43.9 \%)$ of current smokers who visited a health care provider in the past 12 months reported either receiving treatment or receiving a referral to a quit smok- ing program. Of the $43.9 \%$ of smokers who received referrals from a health care provider, $37.5 \%$ were recommended medications and $24.8 \%$ were referred to a quit smoking program. Among those who were referred to a program, only $10.1 \%$ reported that they were assisted by a health care provider to access the program.

Among current smokers, there was no statistically significant difference by age, gender, education or income in regard to whether a health care provider asked about or advised them on smoking. There were few demographic differences in receiving referrals for assistance to quit smoking. Young adult smokers (18 - 24 year olds) reported the lowest rate of referrals compared to $45-64$ year olds who reported the highest rate $(32.7 \%$ vs. $50.4 \%$, $\mathrm{p}<0.05$ ). The likelihood of patients reporting an intervention by a health care provider did not vary by insurance status.

\subsection{Health Status and Reported Interventions}

There was no association between self-reported health status and being asked about smoking. However, there were some significant differences between self-reported health status and being advised not to smoke and reported rates of referral. Smokers who reported their health status as "poor" were significantly more likely to be advised to quit as compared to smokers who reported a health status of either "excellent" or "very good" $(83.3 \%$ vs. $64.1 \%$ and $68.6 \%$, respectively, p < $0.05)$. Similarly, smokers who reported their health status as either "fair" or "good" were significantly more likely to report being referred to treatment by their health care provider, compared to smokers who reported their health status as being "excellent" $(52.7 \%$ and $48 \%$ vs. $31.4 \%$ respectively, $\mathrm{p}<0.05$ ). There was little difference between the number of cigarettes smoked and the likelihood of patient's reporting an intervention by a health care provider.

\subsection{Health Care Provider Interventions, Changes over Time}

Figure 1 shows the percent of current smokers who reported receiving the ask, advise and assist/refer intervention by any health care provider during the past 12 months for 2007 and 2010. The rate of patients being asked if they smoke significantly increased between 2007 and 2010, up 7.8 percentage points. However, the rate of patients who reported being advised not to smoke did not increase. The rate of assistance or referral also did not change significantly. There were small non-significant changes from 2007 to 2010 in smokers receiving recommendations for medication or receiving a referral for a quit smoking program (not shown). 
Table 2. Adult Minnesotans with at least one health care provider visit in the past 12 months among current, former, and never smokers by demographic characteristics.

\begin{tabular}{|c|c|c|c|}
\hline \multirow{2}{*}{ Characteristic } & Current Smokers & Former Smokers & Never Smokers \\
\hline & $\%(95 \% \mathrm{CI})$ & $\%(95 \% \mathrm{CI})$ & $\%(95 \% \mathrm{CI})$ \\
\hline Overall & $70.0(66.0-73.8)$ & $87.6(85.4-89.4)$ & $80.7(79.0-82.4)$ \\
\hline \multicolumn{4}{|l|}{ Gender } \\
\hline Male & $61.0(55.3-66.5)$ & $83.2(79.8-86.1)$ & $71.6(68.6-74.5)$ \\
\hline Female & $80.8(75.6-85.2)$ & $92.6(90.1-94.5)$ & $88.5(86.5-90.2)$ \\
\hline \multicolumn{4}{|l|}{ Age } \\
\hline 18 to 24 & $57.6(47.0-67.5)$ & $79.4(56.5$ - 91.9) & $69.0(63.2-74.3)$ \\
\hline 25 to 44 & $66.8(60.2-72.9)$ & $79.5(74.1-84.0)$ & $79.3(76.1-82.2)$ \\
\hline 45 to 64 & $78.2(72.3-83.1)$ & $89.4(86.4-91.8)$ & $84.4(81.9-86.7)$ \\
\hline More than 65 & $87.7(80.0$ - 92.8) & $94.6(92.4-96.1)$ & $90.0(87.1-92.3)$ \\
\hline \multicolumn{4}{|l|}{ Education } \\
\hline Less than HS & $64.9(50.3-77.3)$ & $88.0(77.9-93.8)$ & $70.3(60.4-78.5)$ \\
\hline HS graduate & $61.2(53.9-68.1)$ & $81.8(76.7-86.0)$ & $75.5(71.2-79.3)$ \\
\hline Some college & $77.1(71.9$ - 81.6) & $88.9(85.5-91.5)$ & $78.8(75.5-81.8)$ \\
\hline College or beyond & $78.4(67.3-86.5)$ & $92.8(90.0-94.8)$ & $87.5(85.4-89.3)$ \\
\hline \multicolumn{4}{|l|}{ Income } \\
\hline Less than $35 \mathrm{k}$ & $68.8(62.4-74.5)$ & $84.9(79.5-89.1)$ & $71.5(66.8-75.7)$ \\
\hline $35 \mathrm{k}$ to $50 \mathrm{k}$ & $77.2(67.5-84.6)$ & $84.9(78.2-89.7)$ & $80.6(75.6-84.9)$ \\
\hline $50 \mathrm{k}$ to $75 \mathrm{k}$ & $66.9(56.8$ - 75.6) & $88.3(83.3-92.0)$ & $81.6(77.5-85.1)$ \\
\hline More than $75 \mathrm{k}$ & $75.7(66.1-83.3)$ & $90.9(87.6$ - 93.4) & $85.7(83.1-88.0)$ \\
\hline
\end{tabular}

Source: 2010 Minnesota Adult Tobacco Survey.

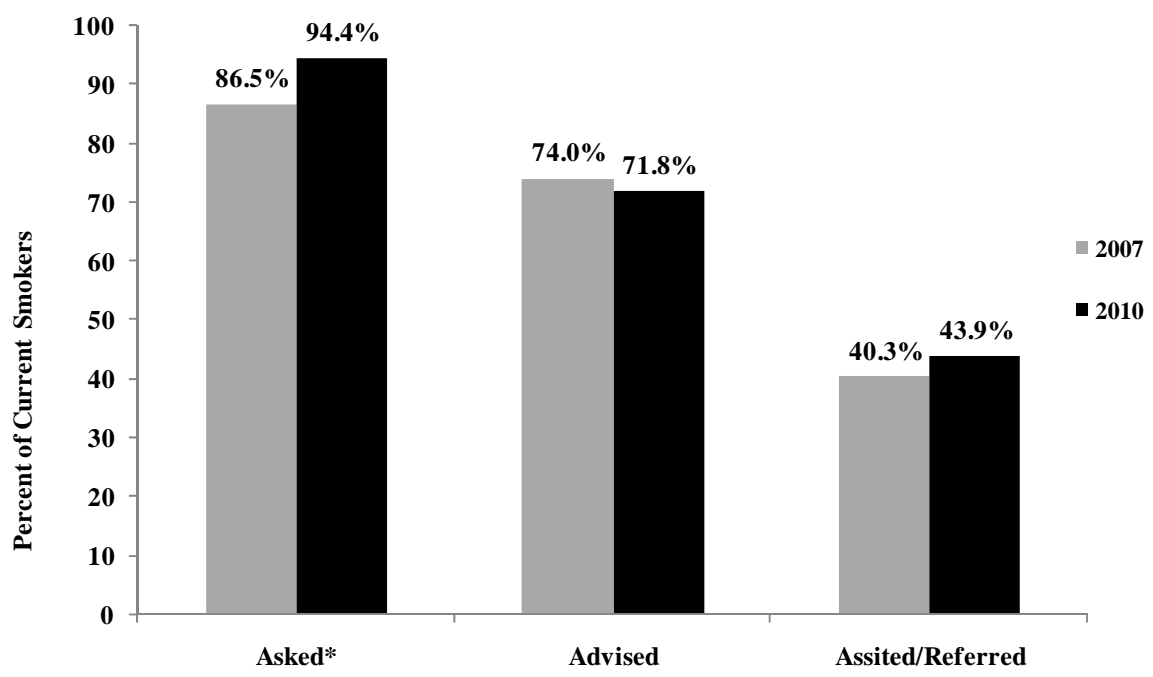

Figure 1. Current smokers who were asked, advised and assisted/referred by a health care provider in the past 12 months, from 2007-2010. Source: Minnesota Adult Tobacco Survey, 2007 and 2010; "Indicates significantly different change from 2007 to 2010 ( $p<0.05)$.

\section{DISCUSSION}

Health care providers are in a unique position to identify smokers among their patients, advise them to quit and assist them by providing medications and referring smokers to quit-smoking programs. Over three-fourths of
Minnesota adults reported at least one health care provider visit over the past 12 months. These findings are consistent with earlier research including the $2009 \mathrm{Na}-$ tional Health Interview Survey that found $81 \%$ of adults made a visit to a doctor or other health professional in the past 12 months, including $74 \%$ of men and $87 \%$ of 
women [2].

The current rate of provider assistance in Minnesota as reported by MATS is also consistent with previous research. A study assessing nine health plans found $80 \%$ of current smokers reported they had visited their clinician in the previous year and of those, $76.6 \%$ reported they were advised to quit, almost two-thirds were assessed for their interest in quitting, $40.6 \%$ were offered a referral to a class or counseling and about one-third were offered pharmacotherapy [8]. Other researchers have found rates of advice to quit ranging from $61 \%$ to $69 \%[9,10,15]$.

Although we found that health care providers do not vary their advice based on the number of cigarettes smoked or insurance status, there were differences based on patient self-reported health status. This is consistent with other studies that have found smokers with the poorest health status were more likely to report ever receiving smoking cessation advice $[9,10]$.

While the reported rate of being asked if they smoke increased between 2007 and 2010, Minnesota smokers who saw a provider during the past 12 months did not report a significant increase in being advised to quit smoking or referred to a quit smoking program. Our findings suggest that about a quarter of smokers $(24.8 \%)$ who received a referral were referred to a quit smoking program and only $10 \%$ of those smokers received help accessing the program. These findings are contrary to what was expected in Minnesota given the evidence supporting the Guideline, the expanding use of EMRs and the availability and promotion of cessation services such as telephone quitlines.

Several studies have looked at impediments to providers implementing smoking cessation interventions, and the most common barrier identified is lack of time [16-18]. Other barriers include lack of skills or confidence [18] and lack of availability of systems for tracking and providing preventive care or proper resources to do so $[17,18]$. Such barriers form the basis of the clinical inertia paradigm - the resistance among clinicians to intensify treatment. In a recent paper, Rindal and colleagues explored clinical inertia as a basis for the treatment of tobacco using dental patients [19].

To further increase rates of smoking cessation, additional efforts should examine how responsibilities can be shared among clinical teams and how to integrate cessation interventions into ongoing clinical care. One option is the use of a fax referral program. A recent study found that $96 \%$ of the US state quitlines offer a fax-referral form for providers to refer patients to a quitline [20]. In addition, a few quitlines (4\%) can refer within an EMR [20] and this mechanism will likely grow as electronic systems expand.

There are some limitations to these findings. First, health care provider visits and interventions are self- reported by the survey participant; therefore the potential for retrospective recall errors exists. In addition, the MATS instrument only provides an overall description of the health care provider intervention that occurred at any time in the past year and did not examine whether a health care provider assessed their willingness to make a quit attempt or arranged to address smoking at the next visit. Further, we did not determine the reason for the visit or the type of health care provider involved in the visit, both of which may factor into whether smoking was addressed.

Our findings are similar to other studies showing high rates of health care providers identifying smokers and advising them to quit but not going the extra step in providing them with a referral and assisting them with their next quit attempt. Further research is needed to look at how systems change and current tools, such as EMRs, can lead to more health care providers integrating the $5 \mathrm{~A}$ 's into clinic care.

\section{ACKNOWLEDGEMENTS}

We would like to thank our partners from Westat, Inc. for their expertise and assistance with the data collection and analysis of these findings. In addition, we would like to thank our colleagues at the University of Wisconsin Center for Tobacco Research and Intervention, Dr. Michael Fiore and Dr. Bruce Christiansen, for their review of this manuscript. We would also like to thank Dr. Christine Sheffer from the Sophie Davis School of Biomedical Education for her review and our colleagues at ClearWay Minnesota ${ }^{\mathrm{SM}}$ for their review, Dr. Barbara Schillo and Ms. Paula Keller. The 2010 Minnesota Adult Tobacco Survey was funded by ClearWay Minnesota ${ }^{\text {SM }}$ with partial support from Blue Cross and Blue Shield of Minnesota.

\section{REFERENCES}

[1] US Department of Health and Human Services (2010) How tobacco smoke causes disease: The biology and behavioral basis for smoking-attributable disease: A report of the Surgeon General. US Department of Health and Human Services, Centers for Disease Control and Prevention, National Center for Chronic Disease Prevention and Health Promotion, Office on Smoking and Health, Atlanta.

[2] US Department of Health and Human Services (2010) Vital health statistics, summary of health statistics for adults: National Health Interview Survey, 2009. DHHS Publication No. (PHS) 2011-1577. Series 10, No. 249.

[3] Woods, S.S. and Jaén, C.R. (2010) Increasing consumer demand for tobacco treatments: Ten design recommendations for clinicians and healthcare systems. The American Journal of Preventive Medicine, 38, S385-S392. doi:10.1016/j.amepre.2009.12.003

[4] Fiore, M., Jaen, C., Baker, T., et al. (2008) Treating tobacco use and dependence: 2008 update Clinical Practice Guideline. US Department of Health and Human Services. Public Health Service, Rockville. 
[5] Aveyard, P., Begh, R., Parsons, A. and West, R. (2011) Brief opportunistic smoking cessation interventions: A systematic review and meta-analysis to compare advice to quit and offer of assistance. Addiction, 107, 1066-1073. doi:10.1111/j.1360-0443.2011.03770.x

[6] Davila, E.P., Zhao, W., Byrne, M., et al. (2009) Correlates of smoking quit attempts: Florida Tobacco Callback Survey, 2007. Tobacco Induced Diseases, 5, 10. doi:10.1186/1617-9625-5-10

[7] Kreuter, M.W., Chheda, S.G. and Bull, F.C. (2000) How does physician advice influence patient behavior? Evidence for a priming effect. Archives of Family Medicine, 9, 426-433.

[8] Quinn, V.P., Hollis, J.F., Smith, K.S., et al. (2009) Effectiveness of the 5-As tobacco cessation treatments in nine HMOs. The Journal of General Internal Medicine, 24, 149-154. doi:10.1007/s11606-008-0865-9

[9] Chase, E.C., McMenamin, S.B. and Halpin, H.A. (2007) Medicaid provider delivery of the 5A's for smoking cessation counseling. Nicotine \& Tobacco Research, 9, 1095-1101. doi:10.1080/14622200701666344

[10] Houston, T.K., Scarinci, I.C., Person, S.D. and Greene, P.G. (2005) Patient smoking cessation advice by health care providers: the role of ethnicity, socioeconomic status, and health. American Journal of Public Health, 95, 1056-1061. doi:10.2105/AJPH.2004.039909

[11] Hsiao, C-J., Hing, E., Socey, T. and Cai, B. (2011) Electronic health record systems and intent to apply for meaningful use incentives among office-based physician practices: United States, 2001-2011. NCHS Data Brief, No 79. National Center for Health Statistics, Hyattsville.

[12] Centers for Disease Control and Prevention (2011) Decrease in smoking prevalence-Minnesota, 1999-2010. Mor- bidity and Mortality Weekly Report, 60, 138-141.

[13] ClearWay Minnesota ${ }^{\text {SM }}$ (2008) Creating a Healthier Minnesota: Progress in Reducing Tobacco Use. http://www.mnadulttobaccosurvey.org/

[14] Centers for Disease Control and Prevention (2011) Behavioral Risk Factor Surveillance System: 2009 Summary Data Quality Report.

http://ftp.cdc.gov/pub/Data/Brfss/2009_Summary_Data Quality_Report.pdf

[15] Smith, S.S., Beckley, T. and Fiore, M.C. (2005) Health care provider use of guideline-based smoking cessation interventions: Results from the 2003 Wisconsin Tobacco Survey. Wisconsin Medical Journal, 104, 28-31.

[16] Holtrop, J.S., Malouin, R., Weismantel, D. and Wadland, W.C. (2008) Clinician perceptions of factors influencing referrals to a smoking cessation program. BMC Family Practice, 9, 18. doi:10.1186/1471-2296-9-18

[17] Gottlieb, N.H., Guo, J.L., Blozis, S.A. and Huang, P.P. (2001) Individual and contextual factors related to family practice residents' assessment and counseling for tobacco cessation. The Journal of the American Board of Family Medicine, 14, 343-351.

[18] Smith, P.M., Sellick, S.M., Brink, P. and Edwardson, A.D. (2009) Brief smoking cessation interventions by family physicians in northwestern Ontario rural hospitals. Canadian Journal of Rural Medicine, 14, 47-53

[19] Rindal, D.B., Rush, W.A. and Boyle, R.G. (2008) Clinical inertia in dentistry: A review of the phenomenon. Journal of Contemporary Dental Practice, 9, 113-121.

[20] North American QUITLINE Consortium (2010) 2010 Survey Data: Results from the 2010 NAQC Annual Survey of Quitlines. http://www.naquitline.org/?page $=$ survey2010 